TH2B-5

\title{
NOVEL TECHNIQUE OF PHASE VELOCITY EQUALIZATION FOR MICROSTRIP COUPLED-LINE PHASE SHIFTERS
}

\author{
Y.C. Leong ${ }^{1}$ and S. Weinreb ${ }^{2}$ \\ ${ }^{1}$ U. of Massachusetts, 619 Lederle GRC, Amherst, MA 01003 \\ ${ }^{2}$ Jet Propulsion Laboratory, 4800 Oak Grove Drive, Pasadena, CA 91109-8099
}

\begin{abstract}
By properly selecting the characteristic impedance and electrical length of the linking line between the coupled lines, phase velocity compensation for microstrip coupled-line phase shifters can be achieved. This technique does not introduce extra element or modification to the coupling in the main coupled lines. It is simple to implement and it has potential to be used in the mm-wave frequencies.
\end{abstract}

\section{INTRODUCTION}

Broadband phase and amplitude balance can be achieved by using various configurations of coupled-line phase shifters [1]. One interesting configuration compares the insertion phases of two different pairs of coupled-line structures to achieve broadband differential phase shift. These coupled-line structures are similar to the one illustrated in Figure 1. This type of phase shifter can be used to synthesize arbitrary differential phase shift that is broadband, compact and totally planar. Full W-band, 180-degree differential phase shift has been demonstrated with this type of structure [2]. However, when the coupledline structures are implemented in microstrip form, the match of the coupled lines becomes frequency dependent and is a function of the odd and even mode phase velocities [3].

Schiek and Köhler [4] had proposed a solution to this problem by using multiple cascaded sections of coupled lines in place of the original coupled lines. As the number of cascaded sections increases, they introduce step discontinuities and this may not be desirable at mm-wave frequencies.

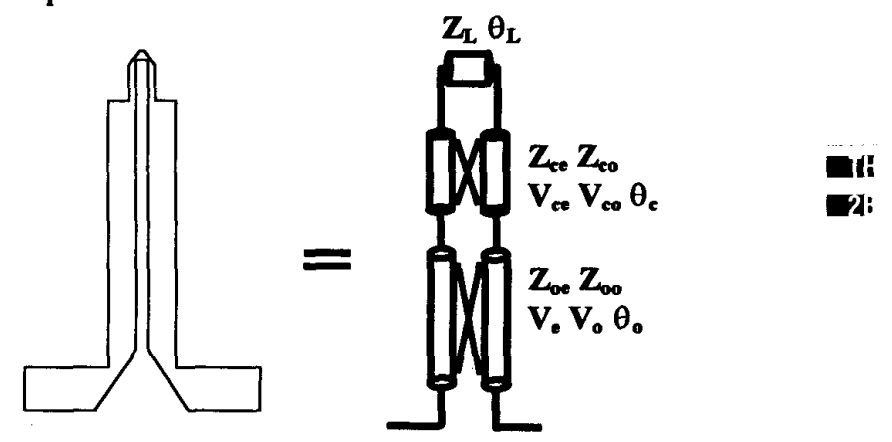

Figure 1. Layout and model for one of the two pairs of coupled lines used in the compensated microstrip coupled-line phase shifter.

In this paper, the influence of the linking transmission line connecting the coupled lines will be analyzed. This effect has often been neglected in the previous works. From the analysis, it is shown that by properly selecting the characteristic impedance and length of the linking transmission line, phase velocity compensation can be achieved at a designed frequency. This technique is simple to implement and it does not introduce extra element or modification to the coupling in the main coupled lines. The compensated microstrip coupled-line phase shifters are broadband, well matched, compact and totally planar. They will be suitable to be implemented as phase bits in switched-type, monolithic mm-wave phase shifters. As compared to the more common reflection-type phase shifters which rely on DC bias to tune for the phase shift in the mm-wave frequencies, the coupled-line phase shifters should provide better temperature stability on the phase and amplitude response. 
To validate the theory of phase velocity compensation, a 4-8 GHz, 90-degree microstrip coupled-line phase shifter had been designed and fabricated on $\mathrm{Er}=10.2$ substrate using this technique.

\section{THEORY}

Figure 1 shows the configuration for one of the two pairs of coupled lines used in the compensated, coupled-line phase shifter. Under even mode excitation at the designed frequency, the linking transmission line (coupled and uncoupled) can be represented as an equivalent transmission line with characteristic impedance equals to $Z_{o e}$ of the main coupled lines and an electrical length of $\theta$ eqe:

$$
\text { beqe }=\frac{\pi}{2}-\tan ^{-1}\left(\frac{\frac{Z_{\alpha}}{Z_{\alpha e}}\left(Z_{L} \cot \frac{\theta_{L}}{2}-Z_{\alpha} \tan \left(\frac{V_{\alpha}+V_{\infty}}{2 V_{\alpha}} \theta_{c}\right)\right)}{Z_{\infty}+Z_{L} \cot \frac{\theta_{L}}{2} \tan \left(\frac{V_{\alpha}+V_{\infty}}{2 V_{\alpha}} \theta_{c}\right)}\right)
$$

where $\theta c$ is the electrical length of the coupled linking transmission line calculated using the average of the odd and even mode phase velocities.

Similarly, under odd mode excitation:

$$
\theta e q o=\tan ^{-1}\left(\frac{\frac{Z_{c o}}{Z_{o c}}\left(Z_{L} \tan \frac{\theta_{L}}{2}+Z_{c o} \tan \left(\frac{V_{c c}+V_{c o}}{2 V_{c o}} \theta_{c}\right)\right)}{Z_{c o}-Z_{L} \tan \frac{\theta_{L}}{2} \tan \left(\frac{V_{c c}+V_{c o}}{2 V_{c o}} \theta_{c}\right)}\right)
$$

When the self-coupling of the linking line is small, an important property can be derived from equations (1) and (2). It can be shown that if the characteristic impedance of the linking line is higher than the geometric mean of the odd and even mode characteristic impedances of the main coupled lines, the odd mode equivalent length will be longer than the even mode equivalent length. This property could be used to equalize the difference in length between the even and odd mode of the main coupled lines.
The difference in length ( $\theta$ eqo- $\theta$ eqe) has been analyzed for GaAs substrate with 100 urn of wafer thickness. Up to 35 degrees of compensating length is achievable for practical values of line width and gap spacing. It is also showed that the range is a function of both the characteristic impedance and coupling of the linking line. Higher characteristic impedance and smaller coupling will give a higher range of compensating length.

\section{DESIGN EQUATIONS}

To exactly compensate for the difference between odd and even mode lengths and to preserve the transmission phase of the coupled lines, the total length of the compensated coupled transmission lines has to be equal to the target length, $\theta_{\text {set }}$ :

$$
\begin{gathered}
\theta_{\text {set }}=\theta_{\text {oo }}+\theta_{\text {eqo }} \\
\theta_{\text {set }}=\theta_{\text {oe }}+\theta_{\text {eqe }}
\end{gathered}
$$

For coupled-line phase shifters, $Z_{o e}, Z_{o o}$ and $\theta_{\text {set }}$ for each of the two pairs of coupled lines are a function of differential phase shift, bandwidth and phase errors. These values can be synthesized using the relations described in [2].

Equation (3) and (4) can be simplified to:

$\theta_{\text {eqo }}-\theta_{\text {eqe }}=\left(\frac{V_{o}}{V_{e}}-1\right)\left(\theta_{\text {set }}-\theta_{\text {eqo }}\right)$

By setting $\theta_{c}$ as the only variable in the above equation, it can easily be solved graphically or numerically. Other variables can be assigned with some practical values based on layout constraints.

Several 90-degree maximally flat phase shifters are designed at $90 \mathrm{GHz}$ and tabulated in Table 1 . The initial design parameters used for the phase shifters are described in [2] and the compensating linking lines are designed using equations (l)-(5). 
Three different types of substrate with relative permittivity of 10.0, 12.4 and 13.0 are used. The thickness of the three substrates is assumed to be $100 \mathrm{um}$ and the thickness of the metal is assumed to be 3 um for all cases. LineCalc (by HP) is used to calculate the microstrip-coupled transmission line parameters at $90 \mathrm{GHz}$.

Free's expression [3] had been used to calculate the match of the compensated coupled lines over frequency by assuming that the frequency dispersion of the phase velocities to be negligible. The frequency response of return loss for all the cases listed in table 1 is similar to that of a lowpass filter and it starts to degrade after passing the designed frequency. The bandwidth performance can be characterized by a corner frequency at which the mismatch becomes significant. A $20 \mathrm{~dB}$ return loss had been used as criteria for evaluating the corner frequencies of the compensated phase shifters. In all designs, the corner frequencies were computed by normalizing them with the designed frequency at $90 \mathrm{GHz}$ and they are better than 1.37 .

\section{EXPERIMENTAL VERIFICATION}

A 4-8 GHz, non-maximally flat 90-degree microstrip coupled-line phase shifter was fabricated using a substrate of relative permittivity of 10.2 and of height $1.27 \mathrm{~mm}$. The layout was simulated using 3D planar electromagnetic simulator to verify the design. Figure 2 shows the measured return and insertion loss of the long and short paths. Figure 3 shows the amplitude and phase balance of the phase shifter pair. The return loss is better than $18 \mathrm{~dB}$ for both the long and short paths across 1-8 $\mathrm{GHz}$. The measured phase balance is $93 \pm 6$ degrees across 4.3-8.8 $\mathrm{GHz}$ and the amplitude balance is better than $\pm 0.5 \mathrm{~dB}$ across the same range.

The good return loss that is achieved across a wide bandwidth using a substrate of high dielectric constant shows that the compensation technique is suitable for use in this application.

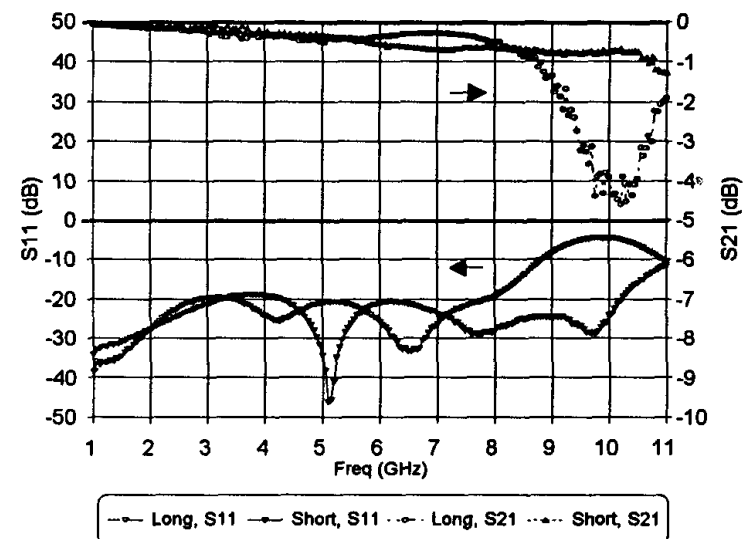

Figure 2. Measured return and insertion loss of the long and short paths of the C-band 90-degree phase shifter.

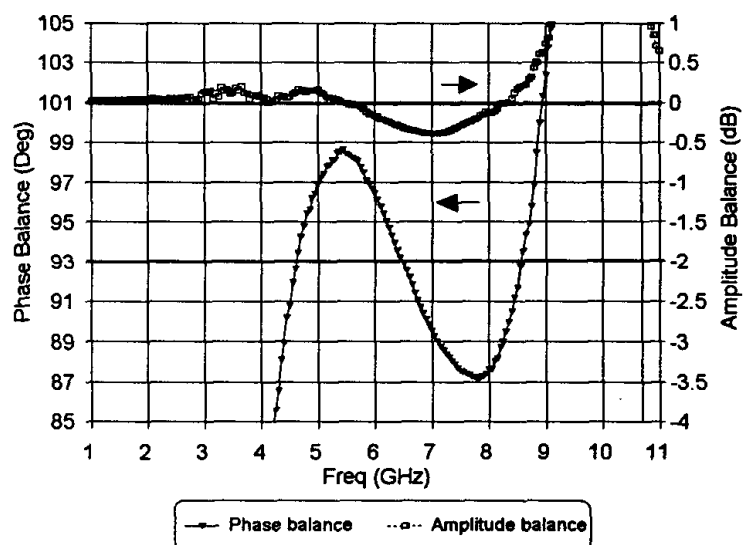

Figure 3. Measured phase and amplitude balance of the long and short paths of the C-band 90degree phase shifter.

The low phase and amplitude balances of the phase shifter indicate that the phase and amplitude errors introduced by the compensation are within reasonable range. The theoretical phase balance of an ideal TEM coupled-line phase shifter is $90 \pm 3$ degrees, with a slightly narrower bandwidth. The simulated phase balance using 3D planar electromagnetic 
simulator is $90 \pm 5$ degrees. This increased in phase error and bandwidth is believed to be due to the contribution of both the compensating line and the microstrip bends.

\section{CONCLUSION}

The 'effect of the linking transmission line on the microstrip coupled-line phase shifters has been analyzed. It has been shown that the linking line can be used to equalize the phase velocities at a designed frequency and thereby improving the match of the coupled-line phase shifters. A 4-8 GHz, 90-degree coupled-line phase shifter had been designed and fabricated using this technique. The return loss for both pairs of coupled lines is better than $18 \mathrm{~dB}$ from 1 to 8 GHz. The amplitude balance is $\pm 0.5 \mathrm{~dB}$ the phase balance is $93 \pm 6$ degrees across $4.3-8.8$ $\mathrm{GHz}$.

\section{REFERENCES}

[1] José Luis Ramos Quirarte and J. Piotr Starski, "INovel Schiffman Phase Shifters," IEEE Trans. Microwave Theory Tech., vol.41, no.1, pp. 9-14, Jan. 1993.

[2] Y.C. Leong and S. Weinreb, "Synthesis of Broaclband TEM Coupled Line Phase Shifters," submitted

[3] Charles E. Free and Colin S. Aitchison, "Improved Analysis and Design of Coupled-Line Phase Shifters," IEEE Trans. Microwave Theory 'Tech., vol.43, no.9, pp. 2126-2131, Sep. 1995.

[4] Burkhard Schiek and Jürgen Köhler, "A Method for Broad-Band Matching of Microstrip Differential Phase Shifters," IEEE Trans. Microwave Theory Tech., vol. 25, no.8, pp. 666-671, Aug. 1977.

\begin{tabular}{|c|c|c|c|c|c|c|c|c|c|c|}
\hline \multirow{2}{*}{ Er } & \multirow{2}{*}{ Path } & \multicolumn{3}{|c|}{ Main C. Line } & \multicolumn{3}{c|}{ Linking C. Line } & \multicolumn{2}{c|}{ Linking Line } & \\
\cline { 3 - 11 } & Zoe & Zoo & $\theta_{\mathrm{o}}$ & Zce & Zco & $\theta_{\mathrm{c}}$ & $\mathrm{Z}_{\mathrm{L}}$ & $\theta_{\mathrm{L}}$ & $\mathrm{f}_{\mathrm{c}}$ \\
\hline 10.0 & Long & 63.6 & 39.3 & 123.7 & 97.4 & 56.6 & 17.8 & 78.5 & 24.0 & 1.39 \\
\hline 10.0 & Short & 63.6 & 39.3 & 92.8 & 97.4 & 56.6 & 2.4 & 78.5 & 24.0 & 1.98 \\
\hline 12.4 & Long & 63.6 & 39.3 & 125.5 & 98.4 & 58.6 & 15.4 & 80.1 & 24.3 & 1.41 \\
\hline 12.4 & Short & 63.6 & 39.3 & 93.4 & 98.4 & 58.6 & 1.4 & 80.1 & 24.3 & 2.00 \\
\hline 13.0 & Long & 63.6 & 39.3 & 122.9 & 96.3 & 57.6 & 17.8 & 78.5 & 25.2 & 1.37 \\
\hline 13.0 & Short & 63.6 & 39.3 & 92.6 & 96.3 & 57.6 & 1.8 & 78.5 & 25.2 & 1.97 \\
\hline
\end{tabular}

Table 1. Design parameters for 90 -degree maximally-flat coupled-line phase shifters with compensation. The designed frequency is centered at $90 \mathrm{GHz}$. 